Konstantina G. Yiannopoulou

Athina Efthymiou

Kleanthis Karydakis

Andreas Arhimandritis

Nikolaos Bovaretos

Mihalis Tzivras

\section{Helicobacter pylori infection as an environmental risk factor for migraine without aura}

Received: 1 August 2007

Accepted in revised form: 8 October 2007

Published online: 17 December 2007

K. G. Yiannopoulou (可) •

A. Efthymiou • K. Karydakis

Department of Neurology,

Laiko General Hospital of Athens,

Athens, Greece

A. Arhimandritis • M. Tzivras •

N. Bovaretos

Department of Gastroenterology and

Pathophysiology, Medical School,

University of Athens, Athens, Greece

K.G. Yiannopoulou

Vas. Tsounia 12A

11526 Maroussi

Athens, Greece

Tel.: +30-2107456592/6932281946

Fax: +30-2107712686

e-mail: ekati2@otenet.gr

\author{
Abstract Helicobacter pylori ( $H$. \\ pylori) infection has recently been \\ associated with various extrain- \\ testinal pathologies and migraine. \\ The aim of this study was to inves- \\ tigate the correlation of the $H$. \\ pylori infection with the pathogen- \\ esis of migraine without aura, \\ especially in cases not affected by \\ endogenous risk factors, like \\ hereditary pattern or hormonal \\ fluctuations. \\ A total of 49 outpatients ( 37 \\ females and 12 males; age range: \\ 19-47 years; mean age: $31, \pm 14$ \\ years) affected by migraine with- \\ out aura was evaluated. We divid- \\ ed them in 2 subgroups: a) with \\ positive familial history, and/or \\ with menstrual type of migraine \\ b) with negative familial history \\ and with menstrual unrelated type \\ of migraine. $H$. pylori infection \\ was diagnosed by the $13 \mathrm{C}$ - urea \\ breath test (INFAI - test). \\ Control subjects consisted of 51 \\ patients without any primary \\ headache history (38 females; \\ mean age of $32, \pm 14,4$ years; range \\ 21-49 years), who underwent \\ upper gastrointestinal (GI) \\ endoscopy for investigation of
}

anaemia or non ulcer dyspepsia. $H$. pylori detection was based on the histologic analysis of gastric mucosa biopsy.

The prevalence of $H$. pylori infection was significantly higher in the migraineurs without aura compared to controls $(p=0.016)$. The prevalence of $H$. pylori infection was significantly high in the mixed and in the female group of our patients without other predisposing factors for migraine without aura ( 81 and $87 \%$ respectively), while in the same groups with predisposing factors (menstruation and/or family history) the prevalence was only 36 and $37 \%$ respectively ( $p=0,001$ for the first group and $p=0,002$ for the second group). Our results seem to highlight the role of $H$. pylori infection as a probable independent environmental risk factor for migraine without aura, especially in patients that are not genetically or hormonally susceptible to migraine.

Keywords Migraine without aura • Helicobacter pylori infection • Hereditary patterns $\cdot$ Menstrual migraine

\section{Introduction}

Occurrence of migraine has been associated with clinically obvious or subclinical extracranial infection (herpes labialis, pharyngitis, cystitis, vaginitis, mycosis and gas- trointestinal inflammation) [1, 2]. It has also been demonstrated that Helicobacter pylori infection has a possible role in precipitation of migraine [3-6], while other data support a simple co-occurrence of $\mathrm{H}$. pylori infection and migraine [7-9].

H. pylori infection is the most common cause of gastri- 
tis and gastric and duodenal ulcers. The association of $H$. pylori infection and various extraintestinal pathologies, such as coronary heart disease, primary Raynaud phenomenon, migraine, Alzheimer's disease and mild cognitive impairment, has recently been addressed [3-6,10-13].

Migraine without aura seems to be caused by a combination of genetic and environmental factors, whereas migraine with aura is probably determined largely by genetic factors $[14,15]$.

The aim of this study was to investigate the correlation of the $H$. pylori infection with the pathogenesis of migraine without aura, especially in cases not affected by endogenous risk factors, like hereditary pattern or hormonal fluctuations.

\section{Materials and methods}

Study design

A total of 49 outpatients (37 females and 12 males; age range: 19-47 years; mean age: $31.6 \pm 14$ years) affected by migraine without aura were evaluated. The cases came from a series of consecutive patients referred to the Neurological Department of the General Hospital of Athens 'Laiko' from January to December 2003. Our study was non-randomised and enrolled the above-mentioned consecutive patients. All patients gave their consent prior to the inclusion in the study and no one refused to participate.

In addition to personal data, participants were asked detailed questions regarding their type of headache: the duration, the frequency, the location, the quality and intensity, the average number of headache attacks per month or per year, as well as the occurrence of nausea, vomiting, photophobia, phonophobia or osmophobia and the occurrence and duration of associated neurological symptoms. Data about relationship with menstruation and hereditary patterns were collected. The lifetime history of migraine for every patient was obtained using the Diagnostic Interview for Headache Syndromes (DIHS), a structured instrument which was developed to collect the major subtypes of headache as defined according to the 2004 International Headache Society (IHS) Criteria for Headache Syndromes [16]. All patients underwent brain CT or MRI without any pathological findings. In all cases $H$. pylori infection was diagnosed by the INFAI test, a non-invasive, simple, highly sensitive and specific method to assess $H$. pylori infection [5].

Migraine was classified as migraine with or without aura according to IHS criteria [9] and as menstrual migraine according to MacGregor's definition, as migraine attacks exclusively starting on the first day of menstruation \pm 2 days and at no other time of the menstrual cycle [17].

Complete pedigrees on first-degree relatives including offspring over 18 years old were obtained for the family of every one of our migraineurs. Face-to-face or telephone assessments of firstdegree relatives, including parents, siblings and offspring $>18$ years were conducted by clinically experienced neurologists who were blind to the diagnostic status of the proband. Information on relatives who were deceased or refused to participate was obtained from multiple relatives. Because the intention was to include noninterviewed relatives, it was not possible to discriminate between specific subtypes of migraine (with and without aura). Final diagnostic assessments were made by a panel of experienced clinicians based on all available information.

The familial history was considered as positive if any of the first-degree relatives had a headache history estimated as migraine and was considered as negative if the headache history of all the first-degree relatives were either negative or indicative of another type of primary or secondary headache $[14,15]$.

The total population of our migraine outpatients was divided into cases without aura and cases with aura. We selected only patients without aura (duration: 1-20 years, median: 9) and we divided them in 2 subgroups: (a) with positive familial history, and/or with menstrual type of migraine and(b) with negative familial history and with menstrual unrelated type of migraine.

Control subjects consisted of 51 patients without migraine (38 females; mean age of $32.8 \pm 14.4$ years; range $21-49$ years), who underwent upper gastrointestinal (GI) endoscopy for investigation of anaemia or non-ulcer dyspepsia at the Department of Gastroenterology and Pathophysiology of the University of Athens during the last year, but in whom endoscopy did not reveal any obvious finding. Mean age and gender ratios did not differ between migraineurs and control participants. H. pylori detection was based on the histologic analysis of gastric mucosa biopsy. Face-to-face or telephone assessments of headache history of control subjects were conducted by clinically experienced neurologists who were blind to the biopsy result. Only subjects without any primary headache history were accepted.

Our study was performed in accordance with the Declaration of Helsinki and was approved by the appropriate investigational review board, which is the ethics committee of the Medical School of the University of Athens.

\section{Statistics}

Statistically significant differences between groups were assessed using the $\chi^{2}$ test and Yates correction when appropriate. Statistical significance was accepted as $p<0.05$.

\section{Results}

The INFAI test was positive in $61 \%$ of the total population of our patients (30 out of 49 migraineurs were infected by H. pylori). H. pylori bacteria were histologically present in $37.25 \%$ of our control subjects (19 out of 51 subjects were infected). The prevalence of $H$. pylori infection was significantly higher in the migraineurs ( $p=0.016$, odds ratio: 2.65 , 
95\% CI: 1.18-5.96).

The prevalence of $H$. pylori infection in the total group of patients (males and females), was significantly higher in migrainous patients with negative familial history and negative correlation with menstruation ( $81 \% v s .36 \%, p=0.001$, odds ratio: 7.70, 95\% CI: 2.09-28.33) than migrainous patients with positive familial history and/or menstrual migraine (22 out of 27 patients vs. 8 out of 22 patients were infected) (Figure 1).

Similar findings were observed in the subgroup of female patients, where the prevalence of $H$. pylori infection was respectively $86 \%$ (18 out of 21 women with menstrual unrelated migraine and/or negative familial history were infected) and $37 \%$ (6 out of 16 women with menstrual migraine and/or positive family history were infected) $(p=0.002$, odds ratio: 10.00 , 95\% CI: 2.04-48.80) (Figure 2).

We also found a higher prevalence of the infection in male patients with negative family history. Among 6 men without family history, 4 were infected, whereas among 6 men with family history, 2 were infected ( p Yates $=0.564$ ).

\section{Discussion}

The prevalence of $H$. pylori infection was significantly higher in the migraineurs without aura compared to controls $(p=0.016)$.

Compared with migraineurs with menstrual migraine and/or positive family history, migraineurs with menstrual unrelated migraine and negative family history were significantly more frequently infected by $H$. pylori.

The prevalence of $H$. pylori infection was significantly high in the mixed and in the female group of our patients without other predisposing factors for migraine without aura (81 and $87 \%$ respectively), while in the same groups with predisposing factors (menstruation and/or family history) the prevalence was only 36 and $37 \%$ respectively. The difference was statistically significant for both the abovementioned groups $(p=0.001$ for the first group and $p=0.002$ for the second group).

The prevalence of infection was also higher in male patients with negative family history, but the size of this
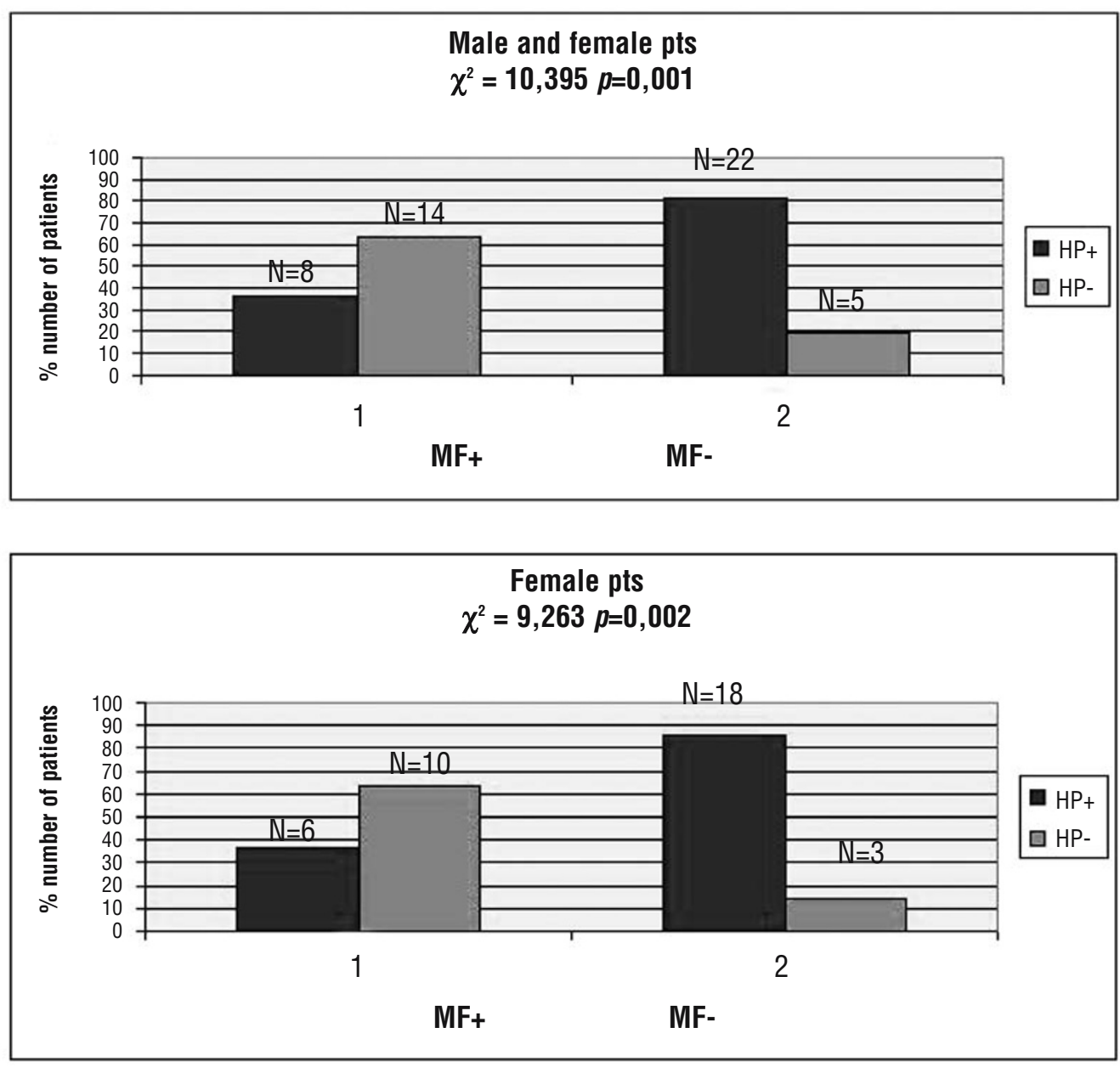

Fig. 1 The prevalence of H. pylori infection in the total group of patients. $\mathrm{HP}+, \mathrm{H}$. pylori infected; $H P-, H$. pylori not infected; $M F+$, patients with menstrual migraine and/or familial history; $M F-$, patients without menstrual migraine or familial history

Fig. 2 The prevalence of $H$. pylori infection in the subgroup of female patients. $\mathrm{HP}+, \mathrm{H}$. pylori infected; $H P-, H$. pylori not infected; $M F+$, patients with menstrual migraine and/or familial history; $M F-$, patients without menstrual migraine or familial history 
subgroup was small.

These results are in agreement with literature data showing higher frequency of $\mathrm{H}$. pylori infection in migraineurs [3-5] and considering that $H$. pylori should be examined in migrainous patients as a possible risk factor that can contribute to clinical improvement of duration and intensity of migraine attacks with its eradication. Consequently, they are in disagreement with other data supporting a simple cooccurrence of $H$. pylori infection and migraine [6-9].

We selected this subgroup of migraineurs (without aura), because this subtype is more often connected with menstrual migraine [18] and we were wondering if there are other significant triggering factors in non-menstrual migraine without aura. But the most important reason for selecting this migraine subgroup was the fact that migraine without aura seems to be caused by a combination of genetic and environmental factors whereas migraine with aura is probably determined largely or exclusively by genetic factors [14, 15]. A previous study reported that spouses to probands with migraine without aura had a slightly increased risk of migraine without aura, while spouses to probands with migraine with aura had no increased risk of migraine with aura [15]. According to our results it is possible that the responsible environmental factor may be $H$. pylori infection.

A significantly higher prevalence of a specific type of $H$. pylori, the CagA-positive H. pylori strains, has been demonstrated only in migraine with aura patients [19]. A plausible mechanism underlying the association between CagA-positive $H$. pylori strains and vascular diseases may be linked to the significantly more intense chronic immuno-inflammatory response that follows colonisation of the gastric mucosa by these strains; cytokines (in particular, interleukin-1, -6, 8 and interferon- $\gamma$ ) and other molecules endowed with proinflammatory, vasospastic and proalgogen properties conceivably may induce a systemic vasculopathy within various arterial districts [10]. We suppose that a similar mechanism produces the association between $H$. pylori infection and migraine without aura but it was impossible to assess the CagA status of $H$. pylori in our patients in that period of time in our hospital, so we cannot conclude anything about this specific topic (CagA-positive strains and migraine without aura). Other recent results do not support the role of oxidative stress in patients suffering from $H$. pylori infection and migraine [20].

Our findings are based on a small sample size and cannot produce consistent results. But we think that they can be useful to clinicians in order to test the possibility of $H$. pylori infection in migraineurs without aura and to researchers as a trigger for larger studies.

Taking into account that hereditary pattern $[14,15]$ and hormonal fluctuations [18] are known and well established endogenous risk factors for migraine, our results seem to highlight the role of $\mathrm{H}$. pylori infection as a probable independent environmental risk factor for migraine without aura, especially in patients that are not genetically or hormonally susceptible to migraine. We suggest that these cases should be more intensively examined for this infection and probably could have the best improvement with its eradication.

\section{References}

1. Chabriat H, Danchot J, Michel P et al (1997) Precipitating factors in migraineurs: a reappraisal in a national control-matched survey. Cephalalgia 17:318-319

2. Covelli V, Pellegrino NM, Jirillo E (2003) A point of view: The need to identify an antigen in psyconeuroimmunological disorders. Curr Pharm Des 9 [suppl 24]:1951-1955

3 Tunca A, Turkay C, Tekin O et al (2004) Is Helicobacter pylori infection a risk factor for migraine? A control study. Acta Neurol Belg 104[suppl 4]:161-164

4. Gasbarrini A, De Luca A, Fiore G et al (1998) Primary headache and Helicobacter pylori. Int J Angiol 7:310-312
5. Gasbarrini A, De Luca A, Fiore G et al (1998) Beneficial effects of Helicobacter pylori eradication on migraine. Hepato-Gastroenterology 45:765-770

6. Gabrielli M, Fiore G, Candelli M et al (2002) Re: "Chronic Helicobacter Pylori Infection and Migraine: A CaseControl Study" (Pinessi L, Savi L, Pellicano R, et al. Headache. 2000;40:836-839). Headache 42[suppl 3]:235-6

7. Ciancarelli I, Di Massimo C, Tozzi Ciancarelli MG et al (2002) Helicobacter pylori infection and migraine. Cephalalgia 22[Suppl 3]:222-225
8. Pinessi L, Savi L, Pellicano R et al (2000) Chronic helicobacter pylori infection and migraine: A case-control study. Headache 40: 836-839

9. Mavromichalis I (2003) The role of Helicobacter pylori infection in migraine. Cephalalgia 23:240

10. Maseri A, Biasucci LM, Liuzzo G (1996) Inflammation in ischaemic heart disease Br Med J 312:1061-1065

11. Gasbarrini A, Serrichio M, Tondi P et al (1996) Association of Helicobacter pylori infection with Raynaud phenomenon. Lancet 348:966-967

12. Kountouras J, Tsolaki M, Gavalas E et al (2006) Relationship between Helicobacter pylori infection and Alzheimer disease. Neurology 28;66:938-940 
13. Kountouras J, Tsolaki M, Boziki M et al (2007) Association between Helicobacter pylori infection and mild cognitive impairment Eur J Neurol 14:976-982

14. Russell MB (2007) Genetics in primary headaches. J Headache and Pain 8:190-195

15. Russell MB, Olesen J (1995) Increased familial risk and evidence of genetic factor in migraine. Br Med J 26;311:541-544
16. Headache Classification Committee of International Headache Society (2004) The International Classification of Headache Disorders, 2nd edn. Cephalalgia 24[Suppl 1]:1-160

17. MacGregor EA (1996) "Menstrual" migraine; towards a definition Cephalalgia 16:11-21

18. Brandes JL (2006) The influence of estrogen on migraine: a systematic review. JAMA 19;295:1824-1830
19. Gasbarrini A, Gabrielli M, Fiore G et al (2000) Association between Helicobacter pylori cytotoxic type I CagA-positive strains and migraine with aura. Cephalalgia 20:561-565

20. Tunca A, Ardicoglu Y, Kargili A, Adam B (2007) Migraine, Helicobacter pylori, and oxidative stress.

Helicobacter 12:59-62 